\title{
The transfer function of the LIF model: from white to filtered noise
}

\author{
Jannis Schuecker ${ }^{1 *}$, Markus Diesmann ${ }^{1,2,3}$, Moritz Helias ${ }^{1,2}$ \\ From The Twenty Third Annual Computational Neuroscience Meeting: CNS*2014 \\ Québec City, Canada. 26-31 July 2014
}

The theory describing correlated activity emerging in recurrent networks relies on the single neuron response to a modulation of its input, i.e. the transfer function. For the leaky integrate-and-fire neuron model exposed to unfiltered synaptic noise the transfer function can be derived analytically $[1,2]$. In this context the effect of synaptic filtering on the response properties has also been studied intensively at the beginning of the last decade $[3,4]$. Analytical results were derived in the low as well as in the high frequency limit. The main finding is that the linear response amplitude of model neurons exposed to filtered synaptic noise does not decay to zero in the high frequency limit. A numerical method has also been developed to study the influence of synaptic noise on the response properties [5]. Here we first revisit the transfer function for neuron models without synaptic filtering and simplify the derivation exploiting analogies between the one dimensional Fokker-Planck equation and the quantum harmonic oscillator. We treat the problem of synaptic filtering with short time constants by reducing the corresponding two dimensional Fokker-Planck equation to one dimension with effective boundary conditions [6]. To this end we use the static and dynamic boundary conditions derived earlier by a perturbative treatment of the arising boundary layer problem [4]. Finally we compare the analytical results to direct simulations (Fig.1) and observe that the approximations are valid up to frequencies in the gamma range $(60-80 \mathrm{~Hz})$. Deviations are explained by the nature of the approximations.

\section{Acknowledgements}

Partially supported by HGF Nachwuchsgruppe VH-NG-1028, the Helmholtz Association: HASB and portfolio theme SMHB, the Jülich Aachen Research Alliance (JARA), the Next-Generation Supercomputer Project of MEXT, EU Grant 269921 (BrainScaleS), and EU Grant 604102 (Human Brain Project, HBP).

\section{Authors' details}

${ }^{1}$ Institute of Neuroscience and Medicine (INM-6) and Institute for Advanced Simulation (IAS-6), Jülich Research Centre and JARA, Jülich, Germany. ${ }^{2}$ RIKEN Brain Science Institute, Wako, Saitama, Japan. ${ }^{3}$ Medical Faculty, RWTH Aachen University, Germany.
A

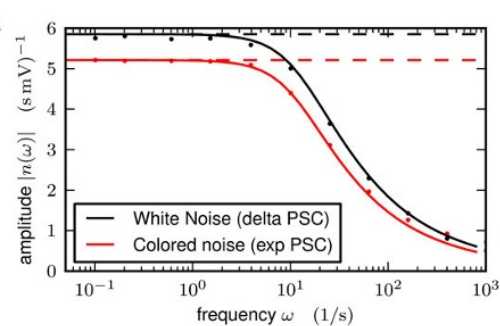

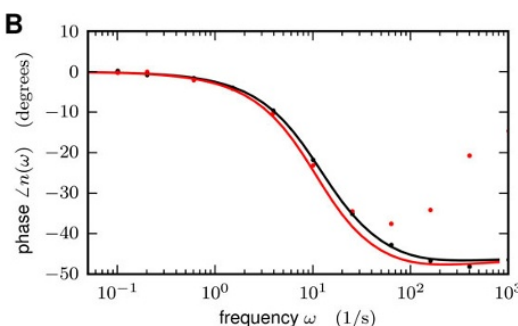

Figure 1 A Linear response amplitude for neurons exposed to colored (red) and white (black) noise. Simulations (dots) and analytical results (curves). B Phase shift of linear response.

\footnotetext{
* Correspondence: j.schuecker@fz-juelich.de

1 Institute of Neuroscience and Medicine (INM-6) and Institute for Advanced

Simulation (IAS-6), Jülich Research Centre and JARA, Jülich, Germany

Full list of author information is available at the end of the article
}

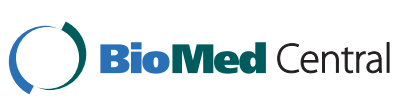

(c) 2014 Schuecker et al; licensee BioMed Central Ltd. This is an Open Access article distributed under the terms of the Creative Commons Attribution License (http://creativecommons.org/licenses/by/4.0), which permits unrestricted use, distribution, and reproduction in any medium, provided the original work is properly cited. The Creative Commons Public Domain Dedication waiver (http://creativecommons.org/publicdomain/zero/1.0/) applies to the data made available in this article, unless otherwise stated. 


\section{References}

1. Brunel N, Hakim V: Fast global oscillations in networks of integrate-andfire neurons with low firing rates. Neural Comput 1999, 11(7):1621-1671.

2. Lindner B, Schimansky-Geier L: Transmission of noise coded versus additive signals through a neuronal ensemble. Phys Rev Lett 2001, 86:2934-2937.

3. Brunel N, Chance FS, Fourcaud N, Abbott LF: Effects of synaptic noise and filtering on the frequency response of spiking neurons. Phys Rev Lett 2001, 86(10):2186-2189.

4. Fourcaud N, Brunel N: Dynamics of the firing probability of noisy integrate-and-fire neurons. Neural Comput 2002, 14:2057-2110.

5. Richardson MJE: Firing-rate response of linear and nonlinear integrateand-fire neurons to modulated current-based and conductance-based synaptic drive. Phys Rev E 2007, 76(2 Pt 1):021919.

6. Klosek MM, Hagan PS: Colored noise and a characteristic level crossing problem. J Math Phys 1998, 39:931-953.

doi:10.1186/1471-2202-15-S1-P146

Cite this article as: Schuecker et al.: The transfer function of the LIF model: from white to filtered noise. BMC Neuroscience 2014 15(Suppl 1): P146.

\section{Submit your next manuscript to BioMed Central} and take full advantage of:

- Convenient online submission

- Thorough peer review

- No space constraints or color figure charges

- Immediate publication on acceptance

- Inclusion in PubMed, CAS, Scopus and Google Scholar

- Research which is freely available for redistribution

Submit your manuscript at www.biomedcentral.com/submit 